\title{
TAFONOMIA COMPARADA DOS CONULATAE (CNIDARIA), FORMAÇÃO PONTA GROSSA (DEVONIANO), BACIA DO PARANÁ, ESTADO DO PARANÁ
}

\author{
SABRINA COELHO RODRIGUES ${ }^{1}$, MARCELLO GUIMARÃES SIMÕES ${ }^{2} \&$ \\ JULIANA DE MORAES LEME ${ }^{1}$
}

\begin{abstract}
COMPARATIVE TAPHONOMY OF CONULATAE (CNIDARIAN), PONTA GROSSA FORMATION (DEVONIAN), PARANÁ BASIN, PARANÁ STATE, BRAZIL A high resolution taphonomic analysis of the Conulatae from the Ponta Grossa Formation, Devonian (Pragian-Emsian), Paraná Basin, Paraná State is presented. The study is based on the occurrences found in rocks that are lithostratigraphically coeval to the Jaguariaíva Member. The taphonomic data gathered indicate that the conulariids [Conularia quichua Ulrich in Steinmann \& Doderlein 1890 and Paraconularia africana (Sharpe 1856)] are preserved according to three distinct taphonomic classes. These classes were established according to: a- three-dimensional distribution of fossils in the matrix; boccurrence of isolated or clustered specimens; $c$ - degree of bioturbation; and d- degree and type of deformation showed by thecae. Taphonomic class I includes isolated and clustered conulariids, vertically oriented to bedding. Commonly, they are inflated, nonfragmented, and preserved in massive or laminated siltstones, sometimes with discrete and isolated ichnofossils. Taphonomic class 2 encompasses isolated or clustered conulariids that are inclined to bedding. Specimens assigned to this class are preserved in intensely bioturbated siltstones or including traces fossils, such as Zoophycus. Taphonomic class 3 is represented by isolated conulariids that are horizontally oriented to bedding, including four subclasses (I to IV) with distinct and complex taphonomic history. Vertically oriented conulariids (class 1) are interpreted as in situ (autochthonous) occurrences, in which mud clouds during storm events abruptly bury living organisms. Inclined (class 2 ) and horizontally oriented conulariids preserved in deeply bioturbated rocks (class 3-I) indicate autochthonous to parautochthonous occurrences. Horizontally oriented conulariids with inflated thecae, found in close association with brachiopod-dominated pavements (i.e., Australospirifer inheringi Kayser 1900) or with hummockies (class 3-II) are interpreted as parautochthonous to allochthonous records. Conversely, horizontally oriented conulariids, that are found incomplete and strongly compressed in massive fine-grained sediments (class 3-III) or in bioturbated siltstones, sometimes with climbing ripples (class 3-IV), record of complex history, including exposure at the sediment/water interface, transport and loss of skeletal hard parts. Conulariids exhibit nonrandom stratigraphic distribution in rocks of the Sequence B. In the geological section of Jaguariaíva, Paraná State, conulariids are preferentially preserved in two well-defined intervals, located at 29 to 32 and at 44 to 48 meters from the top of the Furnas Formation. Layers with in situ occurrences of conulariids (class 1), representing obrution deposits or distal tempestites, located a few centimeters below the marine flooding surfaces characterize these intervals. Finally, the taphonomic data gathered strongly support the idea that the conulariid thecae are not hard structures, but rather are flexible ones and thus able to folding.
\end{abstract}

Keyworls: Conulariids, Taphonomy, Devonian, Ponta Grossa Formation, Paraná Basin.

Resumo Uma análise tafonômica de alta resolução é apresentada para os Conulatae (Cnidaria) da Formação Ponta Grossa, Devoniano (Pragiano-Emsiano), da bacia do Paraná, no Estado do Paraná. O estudo se fundamenta nas ocorrências de conulários de siltitos da Sequiencia B, estratigraficamente correspondentes ao Membro Jaguariaíva. Os dados tafonômicos indicam que os conulários [Conularia cuichua Ulrich in Steinmann \& Doderlein 1890 e Paraconularia africana (Sharpe 1856)] estão preservados segundo três classes tafonômicas, identificadas por 4 critérios: a- atitude em relação ao plano de acamamento; b- se indivíduos isolados ou agrupados; c- grau de bioturbação dos estratos e, d- grau e tipo de deformação apresentado pelas tecas. A classe tafonômica 1 inclui conulários isolados e agrupados, verticalmente orientados. Estão inflados e completos, em siltitos maciços ou incipientemente laminados, às vezes, com icnofósseis isolados, discretos. A classe 2 engloba conulários isolados e agrupados, preservados inclinados, inflados e completos, em siltitos com icnofósseis isolados (p. ex., Zoophycus) ou zonas com generalizada bioturbação. A classe 3 é representada por conulários isolados, horizontalmente orientados, incluindo quatro subclasses (I a IV) com histórias tafonômicas distintas. Os conulários verticais (classe 1) são interpretados como soterrados vivos (in situ), por rápida deposição de sedimentos finos, devido ao aumento brusco nas taxas de sedimentação, associado a eventos de tempestades. Os conulários inclinados (classe 2) ou horizontais, preservados em sedimentos com alto índice de bioturbação (classe 3-l) são também registros autŏctones a parautóctones. Ocorrências de conulários horizontais, com tecas infladas, associados a pavimento de conchas de Australospirifer inheringi Kayser 1900 ou preservados em siltitos com micro hummockies (classe 3-II) são interpretados como registros parautóctones a alóctones. Por sua vez, os conulários horizontais, achatados e incompletos, preservados em folhelhos cinza escuro (classe 3-rII) ou em siltito intensamente bioturbado, com marcas onduladas (classe 3-IV) revelam história tafonômica complexa, com exposição dos restos esqueletais na interface água/sedimento e perda de partes esqueletais. Ao longo da seção de Jaguariaíva, Formação Ponta Grossa, a distribuição vertical dos conulários não é aleatória, pois os mesmos ocorrem em dois intervalos estratigráficos bem definidos, 29 a 32 metros e 44 a 48 metros do topo da Formação Furnas. Tais intervalos incluem depósitos de sufocamento, situados logo abaixo de superfícies de inundação marinha. Finalmente, os dados aqui coligidos suportam, fortemente, a idéia de que a teca dos conulários não foram estruturas rígidas, mas flexíveis e, portanto, passíveis de deformação sem quebra.

Palavras-chave: Conulatae, Tafonomia, Devoniano, Formação Ponta Grossa, bacia do Paraná.

1- Pós-graduação Depto. de Geologia Sedimentar e Ambiental, IGc, USP, Rua do Lago, 562, CEP 18.618.000, São Paulo, SP. scoelho@usp.br, leme@usp.br

2 - CP 510, Lab. Paleozoologia Evolutiva, IB, UNESP, Rubião Júnior, s/n, CEP 18.618 .000 Botucatu, SP. btsimoes@ibb.unesp.br, 
INTRODUÇÃO Conulários são cnidários extintos, bentônicos sésseis, com teca fosfática, piramidal, alongada e, na maioria das vezes, com quatro faces (Van Iten 1991, Van Iten et al. em preparação). São encontrados em todos os tipos de depósitos marinhos (siliciclásticos ou carbonáticos) paleozóiços, de diversas partes do mundo, exceto no continente Antártico (Babcock \& Feldmann 1984). Sua distribuição estratigráfica abrange o intervalo compreendido entre o Neocambriano (Hughes et al. 2000) e o Triássico (McMenamin 1987), embora Ivantsov \& Fedonkin (2002) sugiram possiveis ocorrências de formas aparentadas no Neoproterozóico.

Conulários são elementos comuns no registro fóssil da Formação Ponta Grossa (Devoniano, Pragiano-Emsiano, Grahn et al. 2000, mas vide Bergamaschi \& Pereira 2001, para uma interpretação diferente da idade da Formação Ponta Grossa), da bacia do Paraná, especialmente nos depósitos litoestratigraficamente correspondentes ao Membro Jaguariaíva (Lange \& Petri 1967) ou Seqüência B, do modelo de Bergamaschi (1999) e Bergamaschi \& Pereira (2001). Um aspecto notável dessas ocorrências é o fato de os conulários estarem preservados em depósitos de sufocamento (obrution deposits Simões et al. 2000a, b, c, veja Holz \& Simões 2002, para gênese desses depósitos), ocorrendo in situ, isso é, em posição de vida (Petri \& Fúlfaro 1983, Simões et al. 2000a). Dessa forma, permitem determinar não apenas o modo de vida desses invertebrados, questão ainda em debate entre os paleontólogos (veja discussão em Simões et al. 2000a e Rodrigues, 2002), mas também os processos tafonômico/sedimentares responsáveis por sua preservação.

Tendo em vista o contex to acima, o objetivo do presente trabalho consiste na análise tafonômica básica (sensu Simões \& Holz 2000, Holz \& Simões 2002), com especial atenção para a bioestratinomia de alta resolução (sensu Simões 1998, Simões \& Kowalewski 1998a, b, Simões \& Ghilardi 2000), procurando comprovar a hipótese de que três modos principais de preservação são observados no registro fóssil dos conulários da Formação Ponta Grossa (veja, por exemplo, Simões et al. 2000a). Adicionalmente, visa correlacionar também os dados tafonômicos obtidos com outros sedimentológicos e estratigráficos disponíveis para os depósitos onde os conulários estão preservados, verificando se os padrões de preservação observados variam segundo sua posição estratigráfica na sequiência sedimentar estudada.

MATERIAL EMÉTODOS Geologia da área O material aqui estudado provém de afloramentos da Formação Ponta Grossa, no Município de Jaguariaíva, Estado do Paraná (PR) (Fig. 1). Na área de estudo, estão bem preservados os siltitos do Membro Jaguariaíva (Lange \& Petri 1967) ou da Sequiência B (Bergamaschi 1999. Bergamaschi \& Pereira 2001), referente ao ciclo de sedimentação caracterizado por depósitos marinhos rasos de face praial inferior e costa afora, respectivamente, shoreface e offshore (veja Suguio 1992 para tradução dos termos) (Bergamaschi 1999, Bergamaschi \& Pereira 2001). Em Jaguariaíva, esses estão bem expostos nos clássicos cortes da estrada de ferro JaguariaívaArapoti, desde o $\mathrm{Km} 2,2$ (altitude de $860 \mathrm{~m}$ ) até o Km 6,6 (altitude de $960 \mathrm{~m}$ ), conforme informações em Lange \& Petri (1967). As coletas foram realizadas ao longo de toda a seção, sendo que os conulários ocorrem, preferencialmente, em intervalos com cerca de $4 \mathrm{~m}$ de espessura, isso é, de 29 a $32 \mathrm{~m}$ e de 44 a 48 m do topo da Formação Furnas (Fig. 2). Os pontos de coleta estão, respectivamente, situados próximos aos quilômetros 3,8 (24⒈'05'S/4942'54'W)

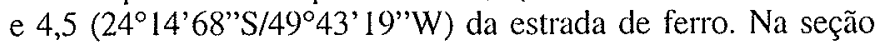
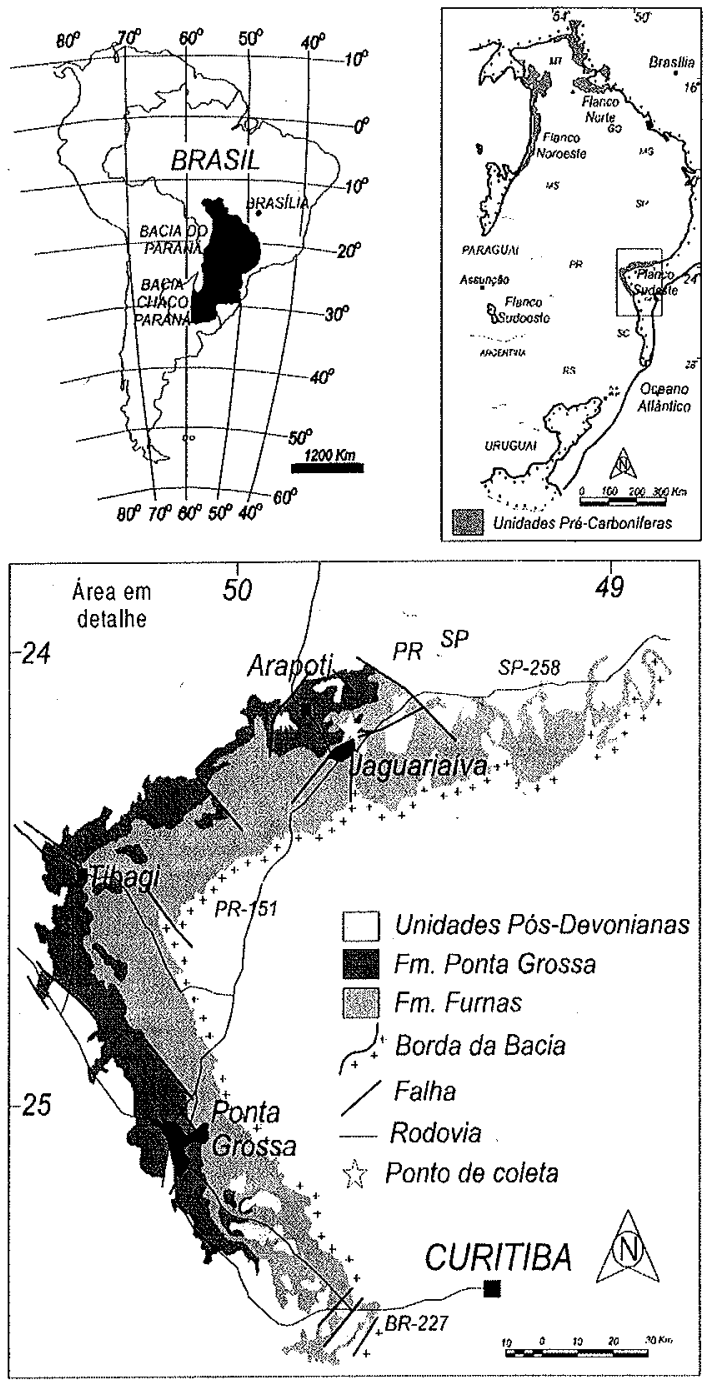

Figura 1 - Mapa de localização das ocorrências fossilíferas es tudadas no Município de Jaguariaiva, Estado do Paraná, PR.

estudada, apenas uma ocorrência foi identificada fora dos intervalos estratigráficos acima referidos, ocorrendo a 39 metros da base da Formação Ponta Grossa. Há ainda menção na literatura sobre raras ocorrências de conulários no topo da seção (Petri 1948 , p. 104). Infelizmente, porém, durante os trabalhos de campo não se logrou êxito em reencontrar tais ocorrências.

Nos intervalos nos quais foi detectada a presença de conulários, predominam os depósitos de sufocamento, os quais são progressivamente sucedidos por folhelho cinza escuro, siltitos e arenitos interlaminados, intensamente bioturbados (Bergamaschi \& Pereira 2001). Os folhelhos escuros correspondem a superfícies de inundação marinha, enquanto os siltitos e arenitos apresentam evidências da ação de ondas ou correntes, portanto, gerados sob condições mais rasas. Conforme será comentado mais adiante a distribuição dos fósseis estudados nos litótipos acima não é ocasional, com importantes implicações estratigráficas e paleoambientais.

Coleta e preparação dos blocos de rochas orientados Os fósseis 


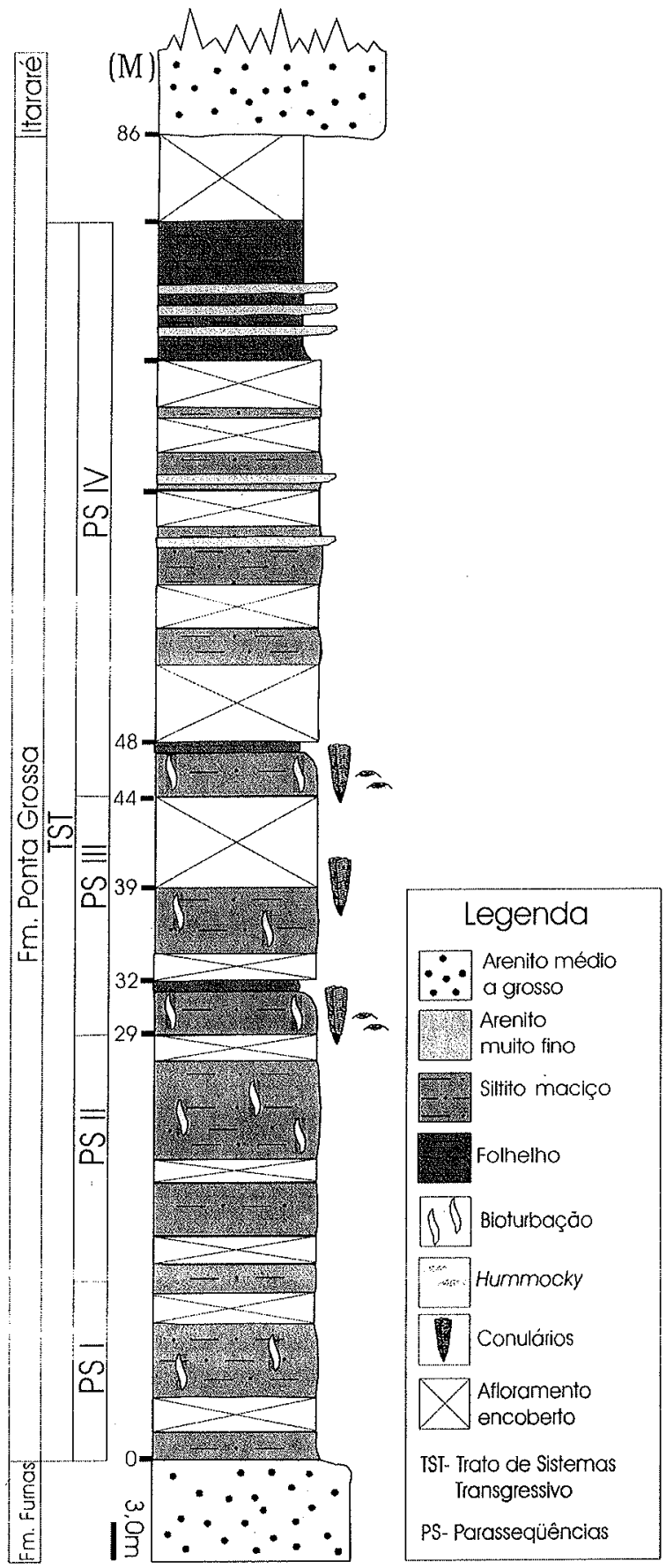

Figura 2 - Seção colunar da Formação Ponta Grossa em Jaguariaiva, PR, modificada de Bergamaschi (1999).

estudados foram coletados segundo a metodologia sugerida por Simões \& Kowalewski (1998a, b), posteriormente ampliada e modificada por Simões \& Ghilardi (2000). Após detalhada inspeção visual, as rochas da parede do afloramento, que continham conulários, foram demarcadas e extraídas na forma de blocos orientados, com volume aproximado de $0,002 \mathrm{~m}^{3}$ (veja Simões \& Ghilardi 2000). Durante os trabalhos de campo, a distribuição vertical das ocorrências de conulários, na sucessão examinada, foi anotada e sua posição, no âmbito da Seqüência B de Bergamaschi
(1999), checada. Uma vez extraídos do afloramento, os 101 blocos retirados (contendo um total de 119 espécimes) foram numerados e preparados de acordo com as técnicas usualmente utilizadas na preparação de macroinvertebrados fósseis (Feldmann et al. 1989). Finalmente, após as diversas etapas de preparação em laboratório, as várias feições sedimentares e tafonômicas presentes nos blocos de rocha foram descritas. Em especial, foram anotadas: a- as orientações dos conulários na matriz sedimentar; b- as estruturas sedimentares presentes tanto junto ao nível de ocorrência dos conulários, como abaixo e acima desses; c-o índice de bioturbação, em porções distintas do bloco (junto, abaixo e acima do nível de ocorrência dos fósseis) e, d- o grau e o tipo de deformação apresentados pelas tecas dos conulários, devidos à compactação que ocorre paralelamente ao seu eixo maior. No geral, as feições tafonômicas observadas e os termos descritivos utilizados seguem Brett \& Baird (1986), Kidwell et al. (1986), Kidwell \& Holland (1991) e Fürsich \& Oschmann (1993). Para a determinação do índice de bioturbação, utilizou-se o método proposto por Miller \& Smail (1997). Todo material aqui estudado está depositado na Coleção Científica do Laboratório de Paleozoologia Evolutiva do Instituto de Biociências, UNESP, campus de Botucatu, sob a sigla DZP.

RESULTADOS Na área de estudo, Formação Ponta Grossa, conulários estão representados, em geral, pelas espécies Conularia quichua Ulrich in Steinmann \& Doderlein 1890 e Paraconularia africana (Sharpe 1856) (Leme 2002). Várias modalidades de preservação (=classes tafonômicas) foram determinados para essas espécies, em parte, compreendendo aqueles já descritos por Simões et al. (2000a). De acordo com os resultados obtidos, a preservação dos conulários varia segundo 4 fatores: a- sua atitude em relação ao plano de acamamento; b- se representado por indivíduos isolados ou agrupados; c- o grau de bioturbação dos estratos e, finalmente, d- o grau e tipo de deformação apresentado pelas tecas. De acordo com essas informações, três classes tafonômicas foram estabelecidas, as quais são resumidamente a seguir descritas.

Classe tafonômica 1 (Fig. 3) É representada por conulários verticalmente orientados, cuja posição dos espécimes pode variar de $90^{\circ}$ a $70^{\circ}$ de inclinação, em relação ao plano de acamamento. Abrange indivíduos isolados ( $\mathrm{n}=21$ espécimes), bem como espécimes agrupados ( $\mathrm{n}=7$ espécimes), em qualquer um dos casos, com a abertura orientada para o topo da camada. As formas agrupadas convergem para um centro comum, em direção à sua região basal. Em todos os casos, os espécimes estão inflados, podendo apresentar algum achatamento longitudinal, que, na maioria das vezes, é mais acentuado na região da abertura da teca. Raros exemplares (p. ex., espécime DZP-3508) apresentam-se comprimidos lateralmente, especialmente na região mediana da teca. Com exceção dos espécimes que ocorrem agrupados, todos os espécimes isolados ocorrem completos, isso é, com as três regiões da teca (região da abertura, mediana e basal) preservadas. Em todos os casos, o material que preenche a cavidade do corpo dos conulários é muito fino, contendo, às vezes, bioclastos representados por conchas isoladas de moluscos bivalves e braquiópodes. Os espécimes dessa classe tafonômica estão preservados tipicamente em siltitos maciços ou incipientemente laminados, às vezes, com icnofósseis isolados, discretos (índice de bioturbação 2, Miller \& Smail 1997), estando presentes acima ou abaixo dos estratos onde os espécimes horizontalmente 
preservados ocorrem.

Classe tafonômica 2 (Fig. 3) É represẹntada por conulários inclinados, cujo eixo maior da teca mostra-se inclinado cerca de $60^{\circ}$ a $20^{\circ}$, em relação ao plano de acamamento. Inclui espécimes isolados ( $n=26$ espécimes) ou agrupamentos ( $n=4$ espécimes) de dois ou três elementos, todos inflados e, às vezes, com achatamento longitudinal ou lateral. Em todos os agrupamentos, os exemplares convergem para um centro comum, em direção à sua região basal, porém na região basal, a teca não está preservada. Na maioria dos casos (indivíduos isolados ou não), a região da abertura dos espécimes está orientada para o topo, em relação ao plano de acamamento. De fato, dentre os 119 espécimes examinados, apenas um não apresenta essa orientação (espécime DZP-17050). Os conulários estão presentes em siltitos com icnofósseis isolados (p. ex., Zoophycus, índice de bioturbação 2) ou zonas de generalizada bioturbação (índice de bioturbação 3, Miller \& Smail 1997).

Classe tafonômica 3 (Fig. 3) É representada por conulários isolados e horizontalmente orientados, formando ângulos de até $20^{\circ}$, em relação ao plano de acamamento. Inclui tanto conulários completos e inflados, como incompletos e achatados. Portanto, essa classe tafonômica engloba 4 subclasses informais, descritas a seguir. A SUBCLASSE I ( $\mathrm{n}=43$ espécimes) é representada por indivíduos inflados ou achatados lateralmente e com as três regiões da teca (abertural, mediana e basal) preservadas, ocorrendo em siltitos intensamente bioturbados (índice de bioturbação 3 ou 4, Miller \& Smail 1997). A SUBCLASSE II ( $\mathrm{n}=8$ espécimes) consiste de indivíduos inflados ou pouco comprimidos lateralmente, com tecas completas, na maioria das vezes. Estão preservados em siltitos ou arenitos muito finos, mostrando estruturas sedimentares, geradas por fluxo oscilatório, tais como as estratificações cruzadas do tipo micro hummocky. Pode haver também associação com pavimentos de conchas desarticuladas de Australospirifer inheringi Kayser 1900. A SUBCLASSE III ( $\mathrm{n}=1$ espécimes) é representada por indivíduos incompletos, com tecas "rasgadas" e muito achatadas lateralmente (faces verdadeiras não são reconhecidas). Os conulários dessa categoria ocorrem em folhelho cinza escuro. Finalmente, a SUBCLASSE IV ( $\mathrm{n}=1$ espécimes) é constituída de espécimes incompletos, faltando a região da abertura e teca fortemente comprimida, isto é, com duas faces adjacentes expostas no mesmo plano de acamamento (espécime DZP-3634). Ocorrem em siltitos intensamente bioturbados (índice de bioturbação 3 ou 4, Miller \& Smail 1997), com marcas onduladas.

Por fim, em qualquer uma das categorias acima, os fósseis que tipicamente ocorrem em associação com os conulários estudados, isso é, na mesma amostra de mão, são os braquiópodes inarticulados (p. ex., Orbiculoidea), os quais retêm também o material de fosfato de cálcio da concha (veja Babcock et al. 1987, para ocorrências similares no Devoniano da Bolívia). Entretanto, fósseis de organismos bentônicos vágeis (p. ex., trilobitas) no mesmo horizonte de ocorrência dos conulários são raros, tendo sido observados, até o momento, sem exceção, somente na ocorrência

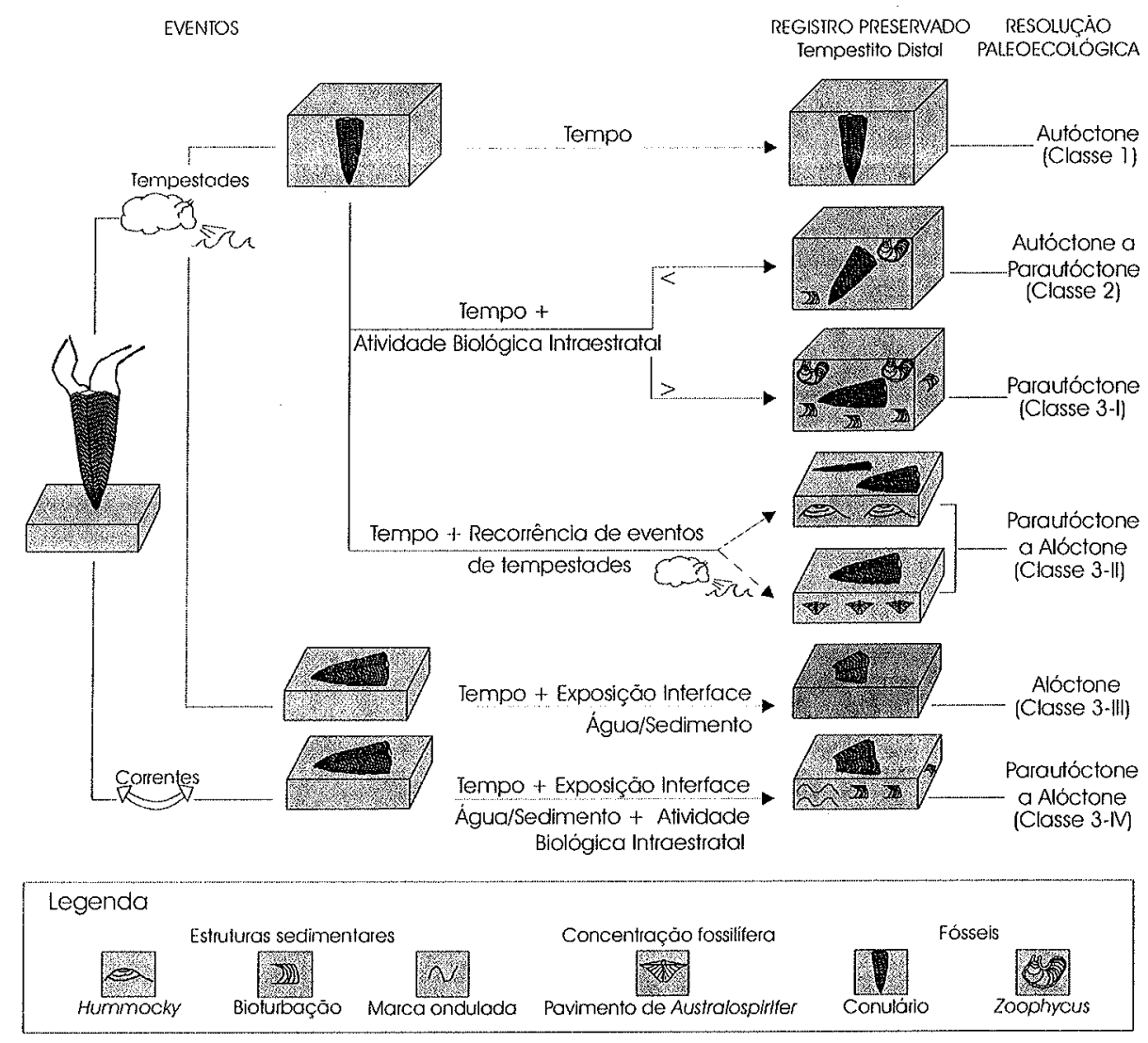

Figura 3 - Modelo tafonômico para conulários da Formação Ponta Grossa. Possíveis trajetórias tafonômicas e a resolução paleoecológica do registro preservado estão também indicadas. 
dos folhelhos cinza escuro (classe tafonômica 3-III).

DISCUSSÃO Análise tafonômica básica As figuras 3 e 4 apresentam uma síntese do modelo tafonômico proposto para as ocorrências dos conulários da Formação Ponta Grossa, região de Jaguariaíva, Estado do Paraná, incluindo as trajetórias tafonômicas inferidas para os registros preservados, bem como seu significado paleoambiental e sua resolução paleoecológica. O esquema básico segue Simões et al. (2000a), acrescido, porém, dos modos: aconulário inclinado, com a abertura voltada para baixo (classe 2); b- conulários horizontais, com tecas parcialmente infladas, preservados em pelito com alto grau de bioturbação (classe 3-I); cconulários horizontais, com tecas comprimidas lateralmente, porém preservando sua tridimensionalidade e associadas a micro hummockies (classe 3-II); d- conulários horizontais, com tecas altamente comprimidas, preservadas em folhelho cinza escuro (classe 3-III) e, e- conulários horizontais, com tecas altamente comprimidas e "rasgadas", preservados em siltitos com alto grau de bioturbação e marcas onduladas (classe 3-IV). De particular importância são as ocorrências in situ, com os conulários preservados em posição de vida e as ocorrências associadas às estruturas sedimentares que indicam atuação de agentes energéticos junto ao fundo. Conforme observado por Bergamaschi (1999) e Bergamaschi \& Pereira (2001), as ondas de tempestades foram o principal agente de transporte de sedimentos dos depósitos aflorantes ao longo da seção de Jaguariaíva, fato já reconhecido por outros autores, a partir de dados tafonômicos (Anelli et al. 1997, Simões et al. 1998a, b, 1999, 2000a, b, c, 2001, 2002, Rodrigues et al. 2000a, b, 2001) e amplamente confirmado nesse estudo.

Possivelmente, o aspecto mais importante a ser considerado diz respeito aos modos de ocorrência e sua relação com o nível de base da bacia (Fig. 4), durante o soterramento final. Ao que tudo indica, os conulários, em especial os da classe tafonômica 1 , foram preservados preferencialmente em depósitos gerados abaixo do nível de base de ondas de tempestade. Por tratar-se de invertebrado séssil de epifauna, sua preservação in situ implica, necessariamente, na ausência de correntes ou fluxos junto ao substrato. Aumento brusco nas taxas de sedimentação, por decantação de finos, em áreas mais distais, durante eventos de tempestades é o principal mecanismo gerador dos chamados depósitos de sufocamento (Miller et al. 1988, Brett \& Seilacher 1991, Brett 1995, Brett et al. 1997). Esses depósitos são formados, entretanto, abai-

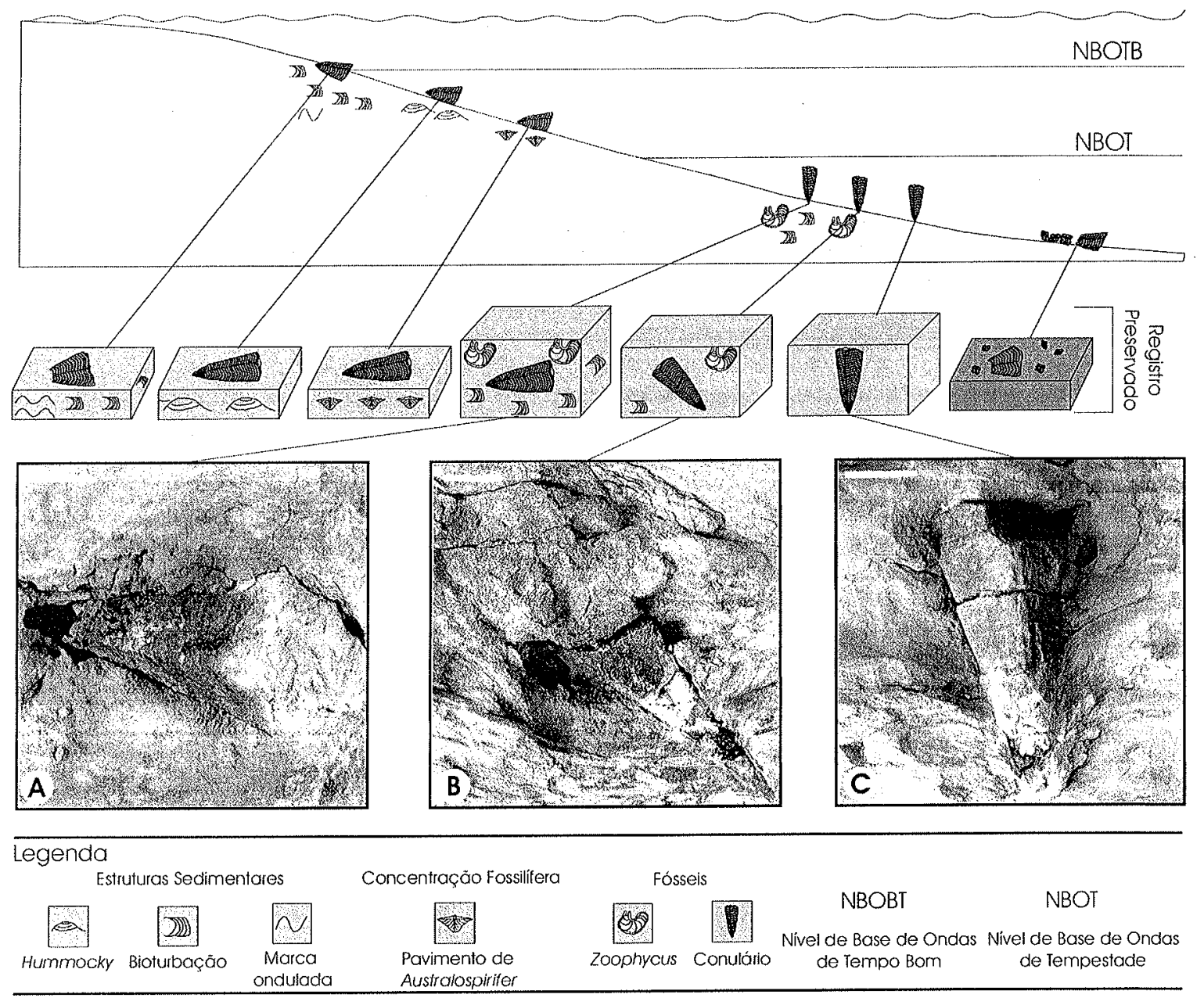

Figura 4 - Distribuição dos conulários ao longo de um gradiente batimétrico de águas rasas/profundas, segundo os dados do Devonaino da bacia do Paraná. A-Conulário horizontalmente orientado, classe tafonômica 3-I (DZP-3329); B-Conulário inclinado, classe tafonômica 2 (DZP-3629); C-Conulário verticalmente orientado, classe tafonômica 1 (DZP-3618). Escala gráfica: lcm. 
xo do nível de ondas de tempestade, possibilitando a preservação da epifauna, em posição de vida (Miller et al. 1988). Desse modo, as ocorrências onde as tecas de conulários estão preservadas junto a estruturas sedimentares do tipo micro hummockies indicam que os conulários foram retirados de sua posição de vida ou dos sedimentos onde estavam preservados, sofrendo curto transporte e rápido soterramento ou sendo redepositados, acima do nível de base de ondas de tempestades.

Embora a tafonomia de conulários seja razoavelmente bem conhecida a partir dos estudos de Harland \& Pickerill (1987) e Simões et al. (2000a, b, c), um aspecto ainda pouco compreendido $\mathrm{e}$, até certo ponto, inexplorado, refere-se à história individual de preenchimento das tecas, por sedimentos, durante o processo de fossilização. Esse aspecto é de grande relevância, não apenas porque permite a preservação tridimensional dessas, mas também por possibilitar um entendimento mais amplo da complexa história de preservação desses invertebrados, no registro sedimentar. Com relação a essa questão, uma ampla variedade de situações é, entretanto, mostrada pelas ocorrências da Formação Ponta Grossa, aqui descritas. Nos casos onde os espécimes estão preservados verticalmente ou inclinados, interpretados como ocorrências in situ, há o predomínio de tecas totalmente infladas, preenchidas por material sedimentar, em geral mais fino do que o da matriz, às vezes. contendo inclusive pequenos fragmentos bioclásticos. Essa situação é compatível com o esperado para os depósitos de sufocamento, onde a decantação de finos e outros materiais colocados em suspensão, durante a passagem de tempestades, acaba soterrando rapidamente os invertebrados bentônicos. Observações experimentais com cifomedusas encalhadas ou mergulhadas em águas saturadas por sedimentos (Bruton 1991, Rozhnov 1998), por exemplo, indicam que o comportamento de bombear intensamente seus canais e cavidades internas resulta no preenchimento dos mesmos por sedimentos, ou seja, seu próprio comportamento de fuga condiciona a sua morte (Hagadorn et al. 2002). Isso explicaria, em parte, como o sedimento poderia ter sido capturado, preenchendo a cavidade gastrovascular dos conulários. Permanecendo nessa condição, os fósseis apresentarão a região da abertura voltada para cima, estando verticalmente preservados, em relação ao plano de acamamento ou inclinados e, até mesmo, horizontais, em decorrência da atividade biológica intraestratal. Tais ocorrências correspondem a registros autóctones a parautóctones (sensu Kidwell et al. 1986), englobando as ocorrências das classes tafonômicas 1, 2 e 3-I. Nessas categorias estão enquadradas a maior parte das ocorrências estudadas.

Aparentemente a teca dos conulários era flexível (vide item implicações paleobiológicals, mais adiante) e após a morte do animal e decomposição das partes moles, a turgidez é perdida. Desse modo, dificilmente a teca permaneceria oca, apta a receber sedimentos, o que torna complicado compreender a história de fossilização das tecas infladas, com compressão desprezível, preservadas horizontalmente junto a estruturas geradas por fluxos oscilatórios, como é o caso das ocorrências da classe tafonômica 3-II. A provável resposta do conulário, em alusão aos cnidários viventes, quando retirados de sua posição de vida, possivelmente, durante as tempestades, e, portanto, passíveis de serem transportados junto ao fundo, seria a contração dos tentáculos e fechamento da região oral. Nessas condições, os sedimentos teriam pouca possibilidade de adentrar na cavidade gastrovascular. Mesmo que tenha ocorrido algum transporte lateral das tecas, dificilmente essas estariam túrgidas, sendo transportadas junto ao fundo, numa mesma direção, possibilitando que as partículas entrassem na cavidade gastrovascular. Em adição, cumpre lembrar que durante as tempestades, que poderiam retirar esses animais de sua posição de vida, o regime de fluxo que predomina é o oscilatório. Novamente, portanto, dificultando imaginar o transporte da teca, junto ao substrato, numa mesma direção, todo tempo. Assim, ocorrências de tecas infladas, com achatamento desprezível, preservadas horizontalmente junto a estruturas geradas por fluxos oscilatórios (classe 3-II) representam, possivelmente, conulários preservados in situ em conseqüiência do aumento brusco nas taxas de sedimentação, associadas à decantação de finos, durante as tempestades. Porém, mudanças no nível de base da bacia, durante tempestades subseqüentes foram, provavelmente, capazes de remobilizar não apenas a fauna vivente, mas também os elementos previamente soterrados, os quais foram redepositados. $O$ achatamento lateral das tecas infladas, observado, por exemplo, no espécime DZP-3624, indica provavelmente que o evento de remobilização ocorreu durante fase na qual os sedimentos no interior das tecas estavam ainda em estado plástico, durante a diagênese. Essas ocorrências representam registros parautóctones a alóctones (sensu Kidwell et al. 1986). Outros exemplos de registros parautóctones a alóctones, que também indicam, possivelmente, o retrabalhamento de elementos já previamente englobados nos sedimentos são os conulários com tecas horizontais e completamente infladas, em associação a pavimentos de conchas de braquiópodes (Australospirifer inheringi) (espécimes DZP-3314, 3315).

Já em fácies de folhelhos cinza escuros, por exemplo, raramente as tecas dos conulários estão preservadas infladas (veja outros exemplos em Van Iten 1991, Van Iten et al. 1996), issoé, guardando sua tridimensionalidade. Em geral, estão completamente achatadas, mostrando a ausência de sedimentos no interior das tecas, durante a diagênese. Isso é fácil de explicar, dada às condições de baixas taxas de sedimentação que predominam nas áreas distais da bacia. Conulários vivendo ou sendo transportados para porções mais distais, deveriam ficar expostos e tombados na interface água/ sedimento. Em decorrência das baixas taxas de sedimentação, a teca não seria completamente preenchida. Em decorrência, o registro mostra tecas fortemente achatadas, com pouco ou nenhum sedimento preenchendo a cavidade do corpo, como é o caso do espécime DZP-17091. Essa ocorrência é um exemplo da classe tafonômica 3-III e representa um registro alóctone (sensu Kidwell et al. 1986). Os conulários da Formação Ponta Grossa, no topo da seção de Jaguariaíva, identificados por Petri (comunicação verbal 2002) e as ocorrências de conulários da Formação Maquoketa (Ordoviciano Superior), da América do Norte, descritas por Van Iten et al. (1996) também constituem exemplos notáveis dessa classe tafonômica.

Finalmente, conulários preservados em fácies indicativas de águas mais rasas, onde estruturas sedimentares resultantes da atuação de fluxos ou correntes junto ao fundo estão presentes, são raros. Isso demonstra que, em condições mais proximais, junto ou acima do nível de base de ondas de tempo bom, o potencial de fossilização é, aparentemente, baixo. Quando presentes, as tecas estão horizontalmente preservadas, muito achatadas lateralmente e "rasgadas", apenas com a região mediana preservada. Essas tecas foram submetidas a transporte e retrabalhamento, possivelmente curto, antes de sua deposição final, constituindo, portanto, registros parautóctones a alóctones (sensu Kidwell et al. 1986). Exemplo dessa situação é a ocorrência da classe 3-IV.

Distribuição paleoambiental Como mencionado, os conulários 
ocorrem em uma ampla variedade de fácies sedimentares, segundo o nível de energia e a sua posição em relação ao nível de base de ondas de tempestade (veja Fig. 4). De uma maneira geral, considerando os dados de literatura (Van Iten 1991, Jerre 1994)e os obtidos para o material da Formação Ponta Grossa, os conulários podem estar preservados em rochas depositadas, sob as seguintes condições e ambientes: a- substratos em águas rasas, plataformais, situados próximos do nível de base de ondas de tempo bom, sujeitos a intenso retrabalhamento. Inclui depósitos formados por arenitos quartzosos, finos a grossos ou calcários bioesparíticos; bsubstratos em águas plataformais, moderadamente profundas, situadas abaixo do nível de base de ondas de tempo bom, sujeitos a atuação de correntes de alta energia, durante tempestades. Abrange depósitos de folhelhos, com arenitos finos e grossos, csubstratos em águas rasas protegidas ou nas porções distais da plataforma, em bacias intracratônicas, abaixo do nível de base das ondas de tempestades. Engloba depósitos de argilitos ou folhelhos pretos, gerados sob condições de baixa oxigenação.

Os dados disponíveis para os conulários estudados indicam que esses ocorrem preferencialmente em siltitos depositados abaixo do nível de base de ondas de tempestades ou entre esse e o nível de base de ondas de tempo bom. Conforme já discutido, as ocorrências in situ indicam a preservação de conulários em posição de vida, em substratos finos, não perturbados por correntes, tracionais. É notável, entretanto, que ao longo da seção estudada. em Jaguariaíva, PR, apenas uma ocorrência tenha sido notada para rochas depositadas sob condições um pouco mais rasas do que aquelas que caracterizam os horizontes com depósitos de sufocamento, que encerram grande quantidade de indivíduos.

Evidências estratigráficas, tafonômicas e anatômicas sugerem, fortemente, que conulários distribuíam-se nos fundos desde o nível de base de ondas de tempo bom até abaixo do nível de base de ondas de tempestade, sendo preservadas em maior número nesse último, graças ao maior potencial de fossilização, sob condiçôes menos energéticas. Embora com estruturas internas nas regiões das arestas e linhas medianas, a teca de Conularia quichua é pouco espessa, dentre as mais delgadas conhecidas para o grupo (Van Iten, comunicação pessoal, 2002). Por outro lado, tecas de Paraconularia africana, que também ocorrem nos siltitos da Formação Ponta Grossa, são mais robustas do que as de C. quichua. Ocorrências de conulários robustos, com tecas e septos fortemente mineralizados, bem como arestas internamente espessadas, em bioesparitos depositados em plataforma carbonática, rasa, foram relatadas por Jerre (1994) para o Siluriano da Suécia. Segundo o autor, o fato de tais espécimes apresentaremse quebrados logo acima da região de inserção dos septos, ou na porção basal, sugere que tais feições de reforço à estrutura da teca constituíram uma adaptação à vida em meio de alta energia. De fato, as únicas ocorrências de tecas infladas e completas de $P$. africana são verificadas em estratos contendo estruturas sedimentares indicativas da atuação de agentes energéticos junto ao fundo, depositados acima do nível de base de ondas de tempestade. Nos horizontes de sufocamento, onde a fauna está preservada in situ, apenas $C$. quichua está presente. Já nos estratos depositados acima do nível de base de ondas de tempestade, fósseis de $C$. quichua são mais raros, quando presentes estão, como visto, achatados e incompletos.

Distribuição estratigráfica Considerando os dados acima e examinando a distribuição vertical dos conulários ao longo da seção de Jaguariaíva (Fig. 2), é notável sua ocorrência em dois intervalos estratigráficos, de aproximadamente 3 a 4 metros, situados, respectivamente, a 29 - 32 metros e a $44-48$ metros do contato com a Formação Furnas. Em outras palavras, os conulários não ocorrem aleatoriamente na seção. Conforme já referido, a Sequiência B (Bergamaschi 1999, Bergamaschi \& Pereira 2001), litoestratigraficamente correspondente ao Membro Jaguariaíva (Lange \&-Petri 1967), compreende um Trato de Sistemas Transgressivo (TST) na concepção de Bergamaschi (1999) e Bergamaschi \& Pereira (2001). Predominam, ao longo da seção, depósitos marinhos-rasos de face praial inferior e costa afora.

Bergamaschi (1999) reconhece ao longo do TST da seção de Jaguariaíva, quatro parassequiências, cuja espessura máxima é da ordem de 20 metros (Fig. 2). Notavelmente, os horizontes contendo conulários em posição de vida ocorrem na base de duas (Simões et al. 2002), das quatro parasseqüências identificadas por Bergamaschi (1999). Esses horizontes correspondem a depósitos de sufocamento. De fato, depósitos de sufocamento parecem ocorrer preferencialmente nos tratos de sistemas transgressivos, na base de parassequiências (Simões et al. 2000b, 2001, 2002), pois nesse intervalo de tempo amplas áreas plataformais, dos mares epicontinentais estão sob condições batimétricas um pouco mais profundas, abaixo do nível de base de ondas de tempestades (Simões et al. 2000b, 2001, 2002). Consequientemente, correntes de fundo raramente afetam o substrato remobilizando os invertebrados da epifauna. Por outro lado, a maior parte da sedimentação deriva da decantação de finos, associados aos eventos de tempestade, que erodem os substratos nas porções mais proximais. Sob tais condições, na média, a exposição dos bioclastos no fundo será reduzida e, graças ao aumento brusco nas taxas de sedimentação em associação com os eventos de tempestades, organismos bentônicos ficarão preservados em posição de vida (Miller et al. 1988, Brett 1995, Brett et al. 1997).

Outro aspecto curioso que pode ser observado é que os poucos conulários que ocorrem nos folhelhos cinza escuro apresentam tecas incompletas e totalmente achatadas, sendo interpretados como registros alóctones. Esses folhelhos apresentam teores de COT (carbono orgânico total) mais elevados do que o da fácies de siltito bioturbado (Bergamaschi, 1999), caracterizando condições de fundos pouco oxigenados, ricos em matéria orgânica em decomposição. Nesses folhelhos, fósseis de moluscos bivalves detritívoros e trilobitas calmonídeos são comuns o que estaria, de acordo com as condições de águas relativamente mais profundas (abaixo do nível de base de ondas de tempestades e fundo rico em matéria orgânica), que devem ter prevalecido durante a sua deposição.

É notável, também, o fato dos fósseis de conulários serem raros nas porções da seção examinada, onde predominam siltitos intensamente bioturbados, depositados sob condições de águas mais rasas, possivelmente dominadas pela ação de ondas ou correntes (Ciguel 1989, Bergamaschi 1999, Bergamaschi \& Pereira 2001). Possivelmente, dada a fragilidade da teca dos conulários, seu potencial de fossilização é baixo, nesses depósitos.

IMPLICAÇÕES PALEOBIOLÓGICAS DOS DADOS TAFONÔMICOS Conulários são invertebrados marinhos, com teca composta por lamelas individuais de fosfato de cálcio (apatita), extremamente finas $(<\mathrm{l} \mu \mathrm{m})$ e paralelas à superfície da teca (Barrande 1867, Boucek \& Ulrich 1929, Moore \& Harrington 1956, Kozlowski 1968, Bishoff 1978, Van Iten 1991, 1992). Tais lamelas reúnem-se em duas camadas, uma interna lisa e outra, externa, ornamentada (Barrande 1867, Slater 1907, Boucek \& Ulrich 1929, Kozlowski 1968, 
Van Iten 1991, 1992). Estudos realizados por Feldmann \& Babcock (1986) com conulários do Mississipiano da América do Norte (Formação Cuyahoga) mostraram que os espécimes apresentavamse parcial ou totalmente comprimidos, especialmente na região da abertura, apresentando teca dobrada, sem indícios de quebra (Feldmann \& Babcock 1986).

De maneira semelhante ao que ocorre com os espécimes da Formação Cuyahoga, alguns dos conulários da Formação Ponta Grossa também apresentam tecas comprimidas. Os conulários das classes tafonômicas 1 e 2 , mesmo inflados, estão achatados longitudinalmente, sendo as dobras da teca mais pronunciadas na região da abertura (Fig. 5). Entretanto, os representantes da classe

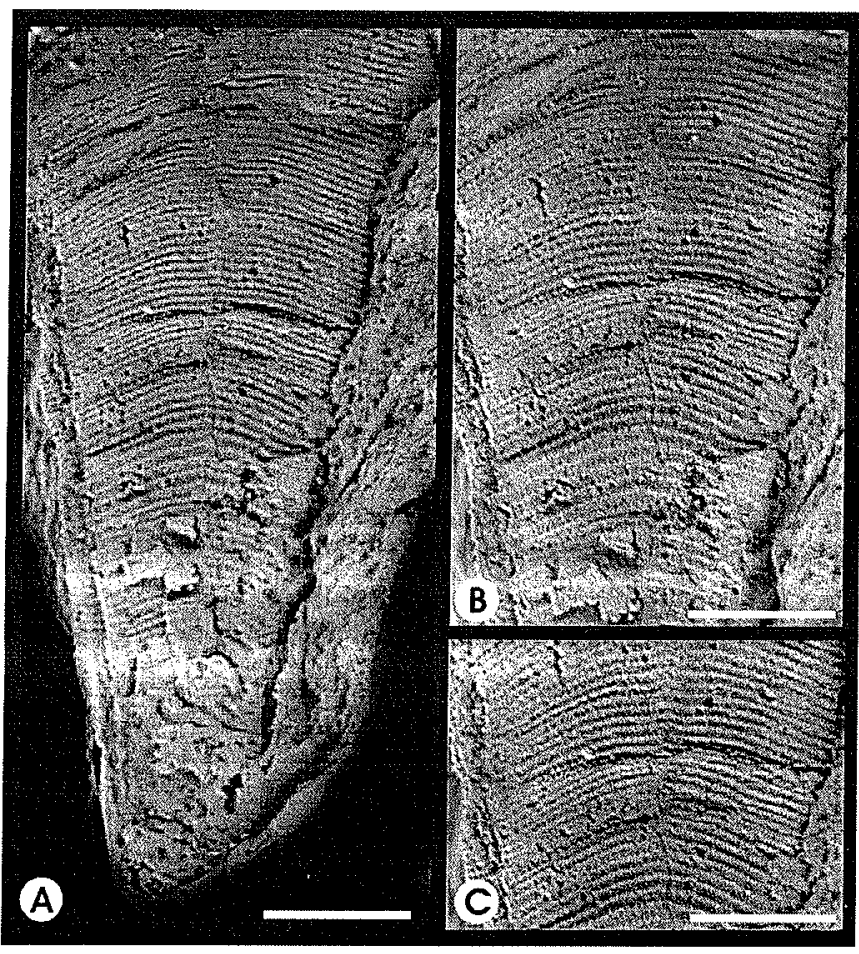

Figura 5-A - Teca de conulário comprimida longitudinalmente (DZP-16814); B-C-Detalhes do mesmo espécime mostrando dobras na região da abertura da teca. Escala gráfica: $0,5 \mathrm{~cm}$.
3 mostram teca com achatamento lateral, seguindo o sentido da compactação da rocha. Em nenhum espécime estudado foram observadas tecas fragmentadas. De fato, as deformações na teca dos conulários indicam que a mesma foi flexível, sendo sustentada pela turgidez intravascular. Esses dados corroboram os de Feldmann \& Babcock (1986), segundo os quais as tecas dos conulários não seriam estruturas tão rígidas quanto conchas de moluscos, por exemplo, mas possivelmente semelhantes à periderme quitinosa de pólipos de coronados (Cnidaria) (Werner 1973, 1979, Van Iten 1991, 1992).

COMENTÁRIOS FINAIS Conulários presentes nos depósitos da Formação Ponta Grossa, na área de Jaguariaíva, Estado do Paraná, são aqui interpretados como animais bentônicos de epifauna, os quais estão preservados, como visto acima, segundo três classes tafonômicas, cujas histórias são bastante complexas. Essas podem ser, em parte reconstituídas a partir de evidências tafonômicas, sedimentológicas e estratigráficas. Na seção de Jaguariaíva, merece destaque o fato de que a distribuição vertical dos conulários e das classes tafonômicas identificadas não é aleatória, já que os mesmos ocorrem em dois intervalos estratigráficos onde depósitos de sufocamento associados às tempestades estão amalgamados. Os resultados obtidos indicam consistentemente que os dados tafonômicos são um importante complemento às análises estratigráficas (vide também Simões et al., 2000b, 2001), especialmente como evidência independente para as interpretações paleoambientais. Por outro lado, os dados tafonômicos contribuem também para o melhor entendimento da paleobiologia desse enigmático grupo de cnidários extintos, mostrando ser essa ciência ferramenta indispensável à análise do registro sedimentar.

Agradecimentos À Dra. M. J. Garcia (UNG), Dra. R. C. T. Cassab (CPRM), Dr. L.E. Anelli (IG/USP) e E. Bosetti (UEPG) por facultar o exame de coleções científicas. Os autores agradecem também o docente Dr. A. C. Marques (IB/USP) e os alunos do programa de pós-graduação do GSA/IG-USP, R. P. Ghilardi, A. M. F. Sales, L. H. C. Mello, F. F. Torello e o Sr. H. A. Rodrigues (IBB/UNESP) pelo auxílio em campo. O texto da versão inicial desse artigo sofreu melhora a partir da leitura crítica dos professores Dr. M. Holz (IG/ UFRGS) e Dr. S. Petri (IG/USP). Aos revisores da RBG pelas sugestões ao manuscrito. A presente pesquisa não teria sido realizada sem o suporte financeiro da FAPESP e do CNPq.

\section{Referências}

Anelii L.E.. Simões M.G., Lucio M.P. 1997. Brazilian bivalves from the Emsian Australocoelia community preserved in situ. Anais Acad. Bras. Ciênc. 69:280-281.

Babcock L.E. \& Feldmann R.M. 1984. Mysterious fossils. Earth Science, $37: 16-17$

Babcock L.E., Feldmann R.M., Wilson M.T., Suárez-Riglos M. 1987. Devonian conulariids of Bolivia. Nat. Geogr: Res., 3:210-231.

Barrande J. 1867. Système Silurien du centre de la Bohême. Iière partie. Tome 3. Classe dês mollueques, ordre dês ptéropodes. Prague, Charlles Bellmann, 179pp.

Bergamaschi S. 1999. Análise estratigráfica do Siluro-Devoniano
(Formação Furnas e Ponta Grossa) da sub-bacia de Apucarana. bacia do Paraná, Brasil. Tese de Doutoramento, Instituto de Geociências, Universidade de São Paulo, 167p.

Bergamaschi S. \& Pereira E. 2001. Caracterização de Seqüências

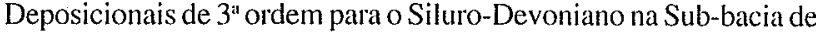
Apucarana, bacia do Paraná, Brasil. In: J.H.G. Melo \& G.J.S. Terra (eds.). Correlações de Seqüências Paleozóicas Sul-Americanas. Ciência Técnica Petróleo, Seção Exploração de Petróleo, pp.: 20-63.

Bishoff G.C.O. 1978. Internal structures of conulariid tests and their functional significance, with special reference to Circonulariina, new suborder (Cnidaria, Scyphozoa). Senck. Lethaia, 59:275-327.

Boucek B. \& Ulrich F. 1929. Étude sur la coquille du genre Conularia 
Miller: Statmiho geologickeho ustavu Ceskoslovenske Republicky, Vestmik, 5:1-25.

Brett C.E. 1995. Sequence, stratigraphy, biostratigraphy, and taphonomy in shallow marine environments. Palaios, 10:597-616.

Brett C.E. \& Baird G.C. 1986. Comparative taphonomy: A key for paleoenvironmental reconstruction. Palaios, 1: 207-227.

Brett C.E. \& Seilacher A. 1991. Fossil Lagerstätten: a taphonomic consequence of event sedimentation. In: G. Einsele, W. Ricken \& A. Seilacher (eds.) Cycles and events in stratigraphy. New York, Berlin, Heidelberg, Springer Verlag, pp.: 283-297.

Brett C.E., Baird G.C., Speyer S.E. 1997. Fossil Lagerstätten: stratigraphic record of paleontological and taphonomic events. In: C.E. Brett \& G.C. Baird (eds.) Paleontological Events: stratigraphic, ecological, and evolutionary implications. New York, Columbia University Press, pp.: $3-40$.

Bruton D.L. 1991. Beach and laboratory experiments with the jellyfish Aurelia and remarks on some fossil "medusoid"traces. In: A.M. Simonetta \& S.C. Momis (eds.) The early evolution of Metazoa and the significance of problematic fossil taxa. Cambridge, Cambridge University Press, pp.: 125-129.

Ciguel J.H.G. 1989. Bioestratigrafia dos Tentaculoidea no flanco oriental da bacia do Paraná e sua ocorrência na América do Sul (OrdovicianoDevoniano). Dissertação de Mestrado, Instituto de Geociências, Universidade de São Paulo, 237p.

Feldmann R.M. \& Babcock L.E. 1986. Exceptionally preserved conulariids from Ohio: reinterpretation of their anatomy. Nat. Geographic Research, 2:464-472.

Feldmann R.M., Chapman R.E., Hannibal J.T. 1989. Paleotechniques. The Paleontological Society, n.4, special Publications, 358pp.

Fursich F.T. \& Oschmann W. 1993. Shell beds as tools in basin analysis: the Jurassic of Kachchh, western India. J.Geol. Soc., 150:169-185.

Grahn Y., Pereira E.. Bergamaschi S. 2000. Silurian and Lower Devonian chitinozoan biostratigraphy of the Paraná Basin in Brazil and Paraguay. Palynology; 24:147-176.

Hagadom J.W., Dott Jr: R.H., Damrow D. 2002. Stranded on a Late Cambrian shoreline: Medusae from central Wisconsin. Geology, 30:147-150.

Harland T.L. \& Pickerill R.K. 1987. Epizoic Schizocrania sp. from the Ordovician Trenton Group of Quebec, with comments on mode of life of conulariids. J. Paleontol., 61:844-849.

Holz M. \& Simões M.G. (Eds.) 2002. Elementos fundamentais de Tafonomia. Porto Alegre, Editora da UFRGS, 240pp.

Hughes N.C.. Gunderson G.O., Weedon M.J. 2000. Late Cambrian conulariids from Wisconsin and Minnesota. J. Paleontol., 74:828838.

Ivantsov A.Y. \& Fedonkin M.A. 2002. Conulariid-like fossil from the Vendian of Russia: a metazoan clade across the Proterozoic/Palaeozoic boundary. Palaeontology, 45:1219-1229.

Jerre F. 1994. Anatomy and phylogenetic significance of Eoconularia loculata, a conulariid from the Silurian of Gotland. Lethaia, 27:97109.

Kalyser, E. 1900. Alguns fósseis paleozóicos do Estado do Paraná. Rev. Museu Paulista, 4:301-311.

Kidwell S.M. \& Holland S.M. 1991. Field discription of coarse bioclaste fabrics. Palaios, 6:426-434.

Kidwell S.M., Fursich F.T., Aigner T. 1986. Conceptual framework for the analysis of fossil concentrations. Palaios, 1:228-238.

Koslowski R. 1968. Nouvelles observations sur lês conulaires. Acta Palaeont. Pol.,13:497-531.

Lange F.W. \& Petri S. 1967. The Devonian of the Paraná Basin. In: J.J. Bigarella (ed.) Problems in Brazilian Devonian Geology. Bol. Paranaense Geoc., 21-22:5-55.

Leme J.M. 2002. Revisão sistemática dos Conulatae Collins et al. 2000, Formação Ponta Grossa, Devoniano (?Lochkoviano-Frasniano), bacia do Paraná, Brasil: implicações paleobiogeográficas e comentários sobre as relações filogenéticas entre os Conulatae. Dissertação de Mestrado, Instituto de Geociências, Universidade de São Paulo, 100p.

McMenamin M.A.S. 1987. The fate of Ediacaran Fauna, the nature of conulariids, and the basal paleozoic predator revolution. In: Geol. Soc. Amer., Abstracts with Programs, 19:29.

Miller K.B., Brett C.E., Parsons K.M. 1988. The paleecologic significance of storm generated disturbance within a Middle Devonian muddy epeiric sea. Palaios, 3:35-52.

Miller M.F. \& Smail S.E. 1997. A semiquantitative field method for evaluating bioturbation on bedding planes. Palaios, 12:391-396.

Moore R.C. \& Harrington H.J. 1956. Scyphozoa. $I n$ : R.C. Moore (ed.). Treatise on Invertebrate Paleontology, Part F, Coelenterata. Geological Society of America, University of Kansas Press, pp.: F54-F66.

Petri S. 1948. Contribuição ao estudo do Devoniano Paranaense. Rio de Janeiro, DNPM/DFPM, 125pp. (Boletim 129)

Petri S. \& Fúlfaro V.J. (Eds.) 1983. Geologia do Brasil: Fanerozóico. São Paulo, EDUSP, 631pp.

Rodrigues S.C. 2002. Tafonomia comparada dos Conulatae Collins, et al. 2000, Formação Ponta Grossa, Devoniano (?LochkovianoFrasniano), bacia do Paraná: implicações paleoeantocológicas e paleoambientais. Dissertação de Mestrado, Instituto de Geociências, Universidade de São Paulo, 100p.

Rodrigues S.C., Leme J.M., Simões M.G. 2000a. Paleoecology of the conulariids (Cnidaria) of the Ponta Grossa Formation (LochkovianFrasnian), from Paraná Basin, Brazil. In: Simpósio Internacional de Iniciação Científica da Universidade de São Paulo, 8, São Carlos, Boletim de Resumos, sem paginação, versão em CD.

Rodrigues S.C., Leme J.M., Simões M.G. 2000b. The role of taphonomy in the taxonomic study of the Devonian Conulatae: some examples from Ponta Grossa Formation (?Lochloviano-Frasniano), Paraná Basin, Brasil. In: SBP/ Núcleo São Paulo, Reunião Anual, Paleo2000/SP, Botucatu, Boletim de Resumos, 15.

Rodrigues S.C., Leme J.M., Simões M.G. 2001. Conularia cf. quichua Uirich: a solitary or clustered marine invertebrate? In: SBP/ Núcleo São Paulo, Reunião Anual, Paleo- 2001/SP, Rio Claro, Boletim de Resumos, 12.

Rozhnov S.V. 1998. Results of burial experiments on the Scyphomedusa Cyanea capilatta L., 1758. Palaeont. Journal, 32:226-228.

Sharpe, D. 1856. Descriptions of Palaeozoic Mollusca from South Africa. Trans. Geol. Soc. London, ser. 2(7):206-215.

Simões M.G. 1998. Tafonomia, time-averaging e resoluç̧ão espacial de concentraçoses fossiliferas intemamente complexas: am estudo de casos do Permiano, bacia do Paraná - Brasil e suas implicaşónes paleocológicas. Tese de Livre-Docência, Instituto de Biociências, Universidade Estadual Paulista, 160p.

Simões M.G. \& Kowalewski M. 1998a. Shell beds as paleoecological puzzles:a case study from the Upper Permian on the Paraná Basin, 
Brazil. Facies, 38: 175-196.

Simōes M.G. \& Kowalewski M. 1998b. Genetic complexity and geobiological implications of "simple" shell beds: an example from the Upper Permian of the Paraná Basin, Brazil. J. African Earth Sciences, 27:179-180.

Simões M.G. \& Ghilardi R.P. 2000. Protocolo tafonômico/ paleautoecológico como ferramenta nas análises paleossinecológicas: exemplos de aplicação em concentrações fossilíferas do Paleozóico da bacia do Paraná, Brasil. Pescuisas em Geociências, 27:3-13.

Simões M.G. \& Holz M. 2000. Tafonomia: processos e ambientes de fossilização. In: I.S. Carvalho (ed.) Paleontologia. Rio de Janeiro, Interciência, pp.: 19-45

Simões M.G., Kowalewski M., Torello F.F., Anelli L.E. 1998a. Longterm time-averaging despite abrupt burial: Paleozoic obrution deposits from epeiric settings of Parana Basin, Brazil. In: Geol. Soc. Amer. Toronto, Abstracts with Prograns: A-384.

Simões M.G., Torello F.F., Kowalewski M., Klein C., Mello L.H.C., Ghilardi R.P. 1998b. Are the Obrution Deposits the most precise and best resolved beds in event stratigraphy? Some Paleozoic examples from the Paraná Basin, Brazil. In: SBG, Cong. Bras. Geol., 40, Belo Horizonte, Anciis, 444.

Simōes M.G., Mello L.H.C., Leme J.M., Rodrigues S.C., Marques A.C. 1999. Devonian conulariid taphonomy and their paleoecological implications. In: Geol. Soc. Amer, Denver, Abstracts with Programs: A-468.

Simões M.G., Mello L.H.C., Rodrigues S.C., Leme J.M., Marques A.C. 2000a. Conulariid taphonomy as a tool in paleoenvirontal analysis. Rev. Bras. Geoc., 30: 757-762.

Simões M.G., Ghilardi R.P., Sales A.M.P., Rodrigues S.C., Leme J.M., Mello L.H.C. 2000b. Taphonomy and genesis of the "Conularia beds" from Ponta Grossa Formation (Devonian) within a sequence stratigraphy framework. In: SBP/ Núcleo São Paulo, Reunião Anual, Paleo-2000/SP, Botucatu, Boletin de Resumos, 20.

Simões M.G., Marques A.C., Collins A.G. 2000c. In situ preservation of conulariids from the Ponta Grossa Formation (Devonian of Brazil), with comments on the phylogenetic placement of Conulatae within Cnidaria. Paleobios, 20:9.

Simões M.G., Ghilardi R.P., Sales A.M.F., Rodrigues S.C., Leme J.M. 2001. Taphonomy and sequency stratigraphic as integrated tools in paleoenvironmental analysis: some examples from the Paraná (Devonian) and Araripe (Cretaceous) Basins, Brazil. $m$ : SBP, Cong. Bras. Paleo., 17, Rio Branco, Boletim de Resumos, 31.

Simões M.G., Ghilardi R.P., Sales A.M.F., Holz M., Rodrigues S.C., Leme J.M. 2002. Taphonomic signatures marking parasequence boundaries in offshore settings: an example from Devonian, Paraná Basin, Brazil. In: Gondwana: correlations and connections, 11, Christchurch, New Zealand, Programme and Abstracts, 78.

Slater I.L. 1907. A monograph of the British Conulariae. Monog. Palacont Soc., 61:1-40.

Steinmann, G. \& Doderlein L. (Eds.) 1890 . Elemente der Paläontologie. Verlag von Wilhelm Engelmann, Leipzig, 848pp.

Suguio, K. 1992. Dicionário de geologia marinha: com termos correspondentes' em inglês, francês e espanhol. T.A. Queiroz, Editor, São Paulo, $171 \mathrm{pp}$.

Van Iten H. 1991. Anatomy, patterns of occurrence, and nature of the conulariid schott. Palaeontology, 34:939-954.

Van Iten H. 1992. Microstructure and growth of the conulariid test: implications for conulariid affinities. Paleontology, 35:359-372.

Van Iten H., Fitzke J.A., Cox R.S. 1996. Problematical fossil cnidarians from the Upper Ordovician of the North-Central USA. Palaeontology, 39: 1037-1064.

Van Iten H., Leme J.M., Rodrigues S.C., Simões M.G. em preparação. New data on the thecal anatomy and biostratinimy of Comularia milwalukeensis Cleland, 1911 and C. quichua Ulrich, 1890 (Devonian, North-Central United States and South-America). J. Paleontol.

Werner B. 1973. New investigations on systematics and evolution of the class Scyphozoa and the phylum Cnidaria. Publs Seto Mar Biol. 20:35-61.

Werner B. 1979. Coloniality in the Scyphozoa: Cnidaria. In: G. Larwood \& B.R. Rosen (eds.) Biology and Systematics of colonial organismls. London, New York, Academic Prees, pp.: 81-103

Manuscrito A-1407

Recebido em 31 de janeiro de 2003

Revisão dos autores em 05 de outubro de 2003 Revisão aceita em 30 de outubro de 2003 